\title{
Blocking both epidermal growth factor receptor and mesenchymal-to-epithelial transition pathways in EGFR-mutated lung cancer
}

\author{
Nagio Takigawa, Nobuaki Ochi, Hiromichi Yamane \\ General Internal Medicine 4, Kawasaki Medical School, Okayama, Japan \\ Correspondence to: Nagio Takigawa. General Internal Medicine 4, Kawasaki Medical School, Okayama, Japan. Email: ntakigaw@gmail.com. \\ Comment on: Wu YL, Zhang L, Kim DW, et al. Phase Ib/II Study of Capmatinib (INC280) Plus Gefitinib After Failure of Epidermal Growth Factor \\ Receptor (EGFR) Inhibitor Therapy in Patients With EGFR-Mutated, MET Factor-Dysregulated Non-Small-Cell Lung Cancer. J Clin Oncol \\ 2018. [Epub ahead of print].
}

Submitted Nov 06, 2018. Accepted for publication Nov 12, 2018.

doi: 10.21037/tlcr.2018.11.01

View this article at: http://dx.doi.org/10.21037/tlcr.2018.11.01

Although most of epidermal growth factor receptor (EGFR) mutant non-small cell lung cancer (NSCLC) is sensitive to EGFR tyrosine kinase inhibitor (TKI), the tumor becomes resistant to the drug after around 1 year. EGFR T790M secondary mutation occupies more than $50 \%$ of the resistant mechanisms. Mesenchymal-to-epithelial transition (MET) amplification is approximately $20 \%$ among non-T790M resistance (1). MET inhibitors include MET selective and non-selective TKIs and anti-MET antibodies such as onartuzumab and emibetuzumab. In the treatment of advanced NSCLC, non-selective TKIs such as tivantinib, cabozantinib, foretinib, crizotinib and glesatinib and selective TKIs such as tepotinib and capmatinib have been developed (2). Although substantial MET expression and MET amplification are observed even in treatmentnaïve NSCLC (1), MET inhibitor monotherapy has not been useful in molecularly unselected NSCLC patients. It is actually effective if the progression of NSCLC depends on MET signals such as MET exon 14 skipping mutations (3).

The reports of MET inhibitor plus EGFR-TKI for the treatment of advanced NSCLC are summarized in Table 1. A randomized phase II study comparing erlotinib plus onartuzumab with erlotinib alone in MET-positive NSCLC, which was determined using immunohistochemistry (IHC), showed improved progression-free survival (PFS) and overall survival (OS) (9). However, the combination in phase III study (METLung) did not show its efficacy (10). Non-selective MET-TKI plus EGFR-TKI have been also investigated. A randomized phase II study of erlotinib with tivantinib showed improved PFS [hazard ratio (HR) 0.18] among patients with KRAS mutations compared with erlotinib monotherapy on exploratory analysis (4). A subsequent phase III study (MARQUEE) demonstrated increased PFS but did not improve OS in the overall nonsquamous NSCLC population (6). Another phase III study in Asian nonsquamous NSCLC patients with wild type-EGFR was prematurely terminated due to the increased interstitial lung disease incidence: 14 (9.2\%, 3 deaths) in the erlotinib plus tivantinib group and 6 (4.0\%, 0 death) in erlotinib plus placebo group (5). The combination group significantly prolonged PFS (HR 0.719 ) but OS was similar (HR 0.891). Previously treated EGFR-mutant NSCLC patients in the MARQUEE study were further analyzed (7). The subanalysis showed that erlotinib plus tivantinib had significantly better median PFS (13.0 vs. 7.5 months for erlotinib monotherapy, HR 0.49) and non-significantly better median OS (25.5 vs. 20.3 months for erlotinib monotherapy, HR 0.68). In 45 EGFR mutationpositive patients who progressed while on EGFR-TKI, the median PFS was only 2.7 months although it was longer in MET high group (4.1 vs. 1.4 months in MET low group) (8). Thus, combination of tivantinib with erlotinib might be effective in previously treated EGFR-mutant and METpositive NSCLC patients.

Crizotinib blocks not only ALK and ROS1 signal pathways but also MET pathway (2). Thus, the combination of crizotinib and EGFR-TKI (erlotinib or dacomitinib) was investigated in phase I studies (Table 1). Crizotinib (300 mg/day) plus erlotinib (100 mg/day), both doses of which were less 


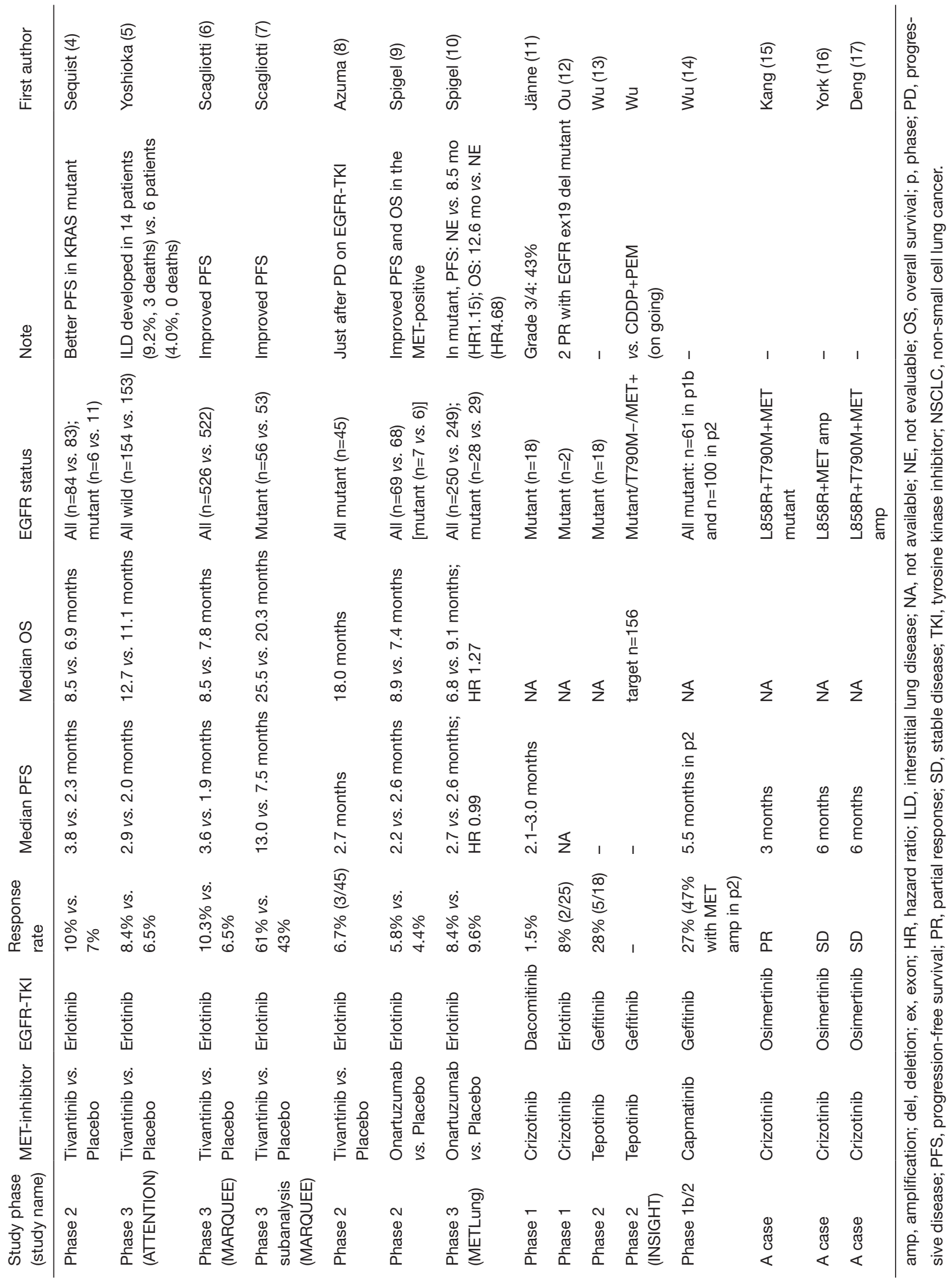


than those approved for single-agent use, was feasible and response was observed in two of 25 patients (12). Both responders had tumors harboring EGFR exon 19 mutation. A phase I study of the combination of crizotinib with second-generation EGFR-TKI, dacomitinib, in NSCLC patients after failure of at least one prior chemotherapy regimen or EGFR-TKI was also reported (11). Only one patient among 33 escalation cohort and 37 expansion cohort achieved objective response. The responding tumor had EGFR exon 19 deletion with T790M mutation and without MET amplification. In the study overall, 66 patients (94\%) had at least one treatment-related adverse events and 27 (39\%) and three (4\%) patients had grade 3 and 4 treatmentrelated adverse events, respectively. The combination was considered to be ineffective and toxic.

How about the combination of MET-selective TKI (capmatinib or tepotinib) with EGFR-TKI? Recently, Wu et al. reported capmatinib plus gefitinib was effective even after failure of EGFR inhibitor therapy in patients with EGFR-mutated and MET amplified NSCLC (14). In the phase $\mathrm{Ib}$ portion $(\mathrm{n}=61)$, the response rate was $23 \%$ regardless of MET status although five patients had EGFR T790M mutation. The patients with EGFR T790M mutation were excluded in the phase II portion. Response was observed in 29 of 100 patients (29\%) regardless of MET status; of the responders, 25 of 29 (86\%) had received an EGFR-TKI as their last prior therapy. The response rate was $47 \%$ in 36 patients whose tumor had high MET gene copy number $(\geq 6)$. The combination seems to be a promising strategy in such EGFR-TKI resistant patients.

Another MET-selective inhibitor, tepotinib, plus gefitinib were also investigated in the 18 Asian patients with METpositive (IHC $3+$ or $2+$ ) and EGFR-mutant NSCLC (13). Partial response was observed in 5 patients and 4 of the 5 responders had high MET-positive (IHC 3+) tumors. Now, tepotinib plus gefitinib are compared with cisplatin plus pemetrexed in advanced NSCLC patients, with acquired resistance to first-line EGFR-TKIs (INSIGHT study, NCT01982955). According to the eligibility criteria on the protocol, the tumor should harbor EGFR-activating mutation without T790M and MET-dysregulated (IHC 2+ or $3+$, amplification, or increased gene copy number). The recruitment was already finished on December 12, 2017 and the estimated study completion date was October 16, 2018. Thus, we are looking forward to seeing the results.

Meanwhile, $40 \%$ of the samples with MET amplification harbored EGFR T790M mutation in EGFR-TKI resistant tumors (1). Inhibition of both MET and EGFR T790M, using MET inhibitor and osimertinib, seems to be attractive strategy. Three patients with EGFR mutation and MET amplification were successfully treated with the combination of crizotinib with osimertinib (15-17). Two of three patients had harboring EGFR L858R and T790M mutations with $M E T$ amplification (15-17). Nowadays, the standard $1^{\text {st }}$ line treatment for EGFR-mutated NSCLC is 3rd generation EGFR-TKI, osimertinib. The resistance mechanisms on progression after $1^{\text {st }}$ line usage of osimertinib have been reported. MET amplification was identified in T790Mpreserved (5/19) and T790M-loss (1/21) cases (18). Seven of 32 osimertinib-resistant tumors (22\%) developed MET amplification (19). In FLAURA study, MET amplification in plasma samples was observed in $15 \%(14 / 19)$ on progression after treatment with $1^{\text {st }}$ line osimertinib (20). Suppressing MET amplification by MET inhibitor combining with osimertinib on $1^{\text {st }}$ line treatment for EGFRmutant advanced NSCLC may more effective, compared with osimertinib monotherapy.

Wu et al. firstly documented that combining selective MET-TKI with EGFR-TKI had substantial effects in EGFRmutated and MET-dysregulated NSCLC on progression after $1^{\text {st }}$ line EGFR-TKI treatment (14). Subsequent randomized studies are warranted. Further, clinical trials of MET-TKI plus EGFR-TKI may be also expected in $1^{\text {st }}$ line treatment in EGFR-mutant NSCLC patients.

\section{Acknowledgements}

None.

\section{Footnote}

Conflicts of Interest: N Takigawa has received research funds and honoraria from AstraZeneca, Boehringer-Ingelheim, Chugai Pharmaceutical, Novartis and Pfizer Inc outside from this work. The other authors have no conflicts of interest to declare.

\section{References}

1. Bean J, Brennan C, Shih JY, et al. MET amplification occurs with or without T790M mutations in EGFR mutant lung tumors with acquired resistance to gefitinib or erlotinib. Proc Natl Acad Sci U S A 2007;104:20932-7.

2. Wu YL, Soo RA, Locatelli G, et al. Does c-Met remain a rational target for therapy in patients with EGFR TKIresistant non-small cell lung cancer? Cancer Treat Rev 
2017;61:70-81.

3. Takigawa N. MET-inhibitors meet MET mutations in lung cancer. Transl Cancer Res 2016;5:S1248-54.

4. Sequist LV, von Pawel J, Garmey EG, et al. Randomized phase II study of erlotinib plus tivantinib versus erlotinib plus placebo in previously treated non-small-cell lung cancer. J Clin Oncol 2011;29:3307-15.

5. Yoshioka H, Azuma K, Yamamoto N, et al. A randomized, double-blind, placebo-controlled, phase III trial of erlotinib with or without a c-Met inhibitor tivantinib (ARQ 197) in Asian patients with previously treated stage IIIB/IV nonsquamous nonsmall-cell lung cancer harboring wildtype epidermal growth factor receptor (ATTENTION study). Ann Oncol 2015;26:2066-72.

6. Scagliotti G, von Pawel J, Novello S, et al. Phase III Multinational, Randomized, Double-Blind, PlaceboControlled Study of Tivantinib (ARQ 197) Plus Erlotinib Versus Erlotinib Alone in Previously Treated Patients With Locally Advanced or Metastatic Nonsquamous NonSmall-Cell Lung Cancer. J Clin Oncol 2015;33:2667-74.

7. Scagliotti GV, Shuster D, Orlov S, et al. Tivantinib in Combination with Erlotinib versus Erlotinib Alone for EGFR-Mutant NSCLC: An Exploratory Analysis of the Phase 3 MARQUEE Study. J Thorac Oncol 2018;13:849-54.

8. Azuma K, Hirashima T, Yamamoto N, et al. Phase II study of erlotinib plus tivantinib (ARQ 197) in patients with locally advanced or metastatic EGFR mutation-positive non-small-cell lung cancer just after progression on EGFRTKI, gefitinib or erlotinib. ESMO Open 2016;1:e00063.

9. Spigel DR, Ervin TJ, Ramlau RA, et al. Randomized phase II trial of Onartuzumab in combination with erlotinib in patients with advanced non-small-cell lung cancer. J Clin Oncol 2013;31:4105-14.

10. Spigel DR, Edelman MJ, O'Byrne K, et al. Results From the Phase III Randomized Trial of Onartuzumab Plus Erlotinib Versus Erlotinib in Previously Treated Stage IIIB or IV Non-Small-Cell Lung Cancer: METLung. J Clin Oncol 2017;35:412-20.

11. Jänne PA, Shaw AT, Camidge DR, et al. Combined PanHER and ALK/ROS1/MET Inhibition with Dacomitinib and Crizotinib in Advanced Non-Small Cell Lung Cancer: Results of a Phase I Study. J Thorac Oncol 2016;11:737-47.

12. Ou SI, Govindan R, Eaton KD, et al. Phase I Results from a Study of Crizotinib in Combination with Erlotinib in Patients with Advanced Nonsquamous Non-Small Cell Lung Cancer. J Thorac Oncol 2017;12:145-51.

13. Wu YL, Soo RA, Kim DW, et al. Tolerability, efficacy and recommended phase II dose (RP2D) of tepotinib plus gefitinib in Asian patients with c-Met-positive/ EGFR-mutant NSCLC: Phase Ib data. J Clin Oncol 2016;34:e20501.

14. Wu YL, Zhang L, Kim DW, et al. Phase Ib/II Study of Capmatinib (INC280) Plus Gefitinib After Failure of Epidermal Growth Factor Receptor (EGFR) Inhibitor Therapy in Patients With EGFR-Mutated, MET FactorDysregulated Non-Small-Cell Lung Cancer. J Clin Oncol 2018. [Epub ahead of print].

15. Kang J, Chen HJ, Wang Z, et al. Osimertinib and Cabozantinib Combinatorial Therapy in an EGFRMutant Lung Adenocarcinoma Patient with Multiple MET Secondary-Site Mutations after Resistance to Crizotinib. J Thorac Oncol 2018;13:e49-53.

16. York ER, Varella-Garcia M, Bang TJ, et al. Tolerable and Effective Combination of Full-Dose Crizotinib and Osimertinib Targeting MET Amplification Sequentially Emerging after T790M Positivity in EGFR-Mutant NonSmall Cell Lung Cancer. J Thorac Oncol 2017;12:e85-8.

17. Deng L, Kiedrowski LA, Ravera E, et al. Response to Dual Crizotinib and Osimertinib Treatment in a Lung Cancer Patient with MET Amplification Detected by Liquid Biopsy Who Acquired Secondary Resistance to EGFR Tyrosine Kinase Inhibition. J Thorac Oncol 2018;13:e169-72.

18. Le X, Puri S, Negrao MV, et al. Landscape of EGFR -dependent and -independent resistance mechanisms to osimertinib and continuation therapy post-progression in EGFR-mutant NSCLC. Clin Cancer Res 2018. [Epub ahead of print].

19. Piotrowska Z, Isozaki H, Lennerz JK, et al. Landscape of acquired resistance to osimertinib in EGFR-mutant NSCLC and clinical validation of combined EGFR and RET inhibition with osimertinib and BLU-667 for acquired RET fusion. Cancer Discov 2018. [Epub ahead of print].

20. Ramalingam SS, Cheng Y, Zhou C, et al. Mechanisms of acquired resistance to first-line osimertinib: Preliminary data from the phase III FLAURA study. Annal Oncol 2018;29:LBA50.

Cite this article as: Takigawa N, Ochi N, Yamane H. Blocking both epidermal growth factor receptor and mesenchymalto-epithelial transition pathways in EGFR-mutated lung cancer. Transl Lung Cancer Res 2018;7(Suppl 4):S352-S355. doi: 10.21037/tlcr.2018.11.01 